\title{
ARTICLE OPEN \\ Stable InSe transistors with high-field effect mobility for reliable nerve signal sensing
}

\author{
Jianfeng Jiang ${ }^{1,2,3}$, Jingxin Li $\mathbb{C}^{4,5}$, Yutao Li $\mathbb{C}^{6}$, Jiazhzhi Duan ${ }^{7}$, Linshen Li ${ }^{6}$, Ye Tian ${ }^{6}$, Zhihua Zong ${ }^{4}$, Haotian Zheng ${ }^{4}$, Xianjin Feng ${ }^{3}$, \\ Qiqiang Li ${ }^{1}$, Hong Liu ${ }^{7,8}$, Yu Zhang ${ }^{1}$, Tian-Ling Ren ${ }^{6}$ and Lin Han ${ }^{1,2}$
}

Among two-dimensional layered semiconductors, indium selenide (InSe) is one of the most promising materials with absolute advantages in field-effect transistors (FETs) because of its high electron mobility and stable material properties. Some work has been performed to improve the mobility of InSe FETs. However, in practical applications, electrical stability of FETs is another essential factor to guarantee the performance of the electronic system. Here, we show a highly stable InSe FET with a field-effect mobility of $1200 \mathrm{~cm}^{2}$ V.s in the practical working regime. The bottom-gate staggered InSe FET was fabricated with a polymethyl methacrylate (PMMA)/HfO 2 dual-layer gate dielectric and PMMA back-channel encapsulation. The hysteresis was maintained at $0.4 \mathrm{~V}$ after 30 days of storage under normal ambient conditions, and the threshold voltage shift was retained at $0.6 \mathrm{~V}$ with a gate stress $V_{\mathrm{GS}}$ of $10 \mathrm{~V}$, which represents the best electrical stability reported to date. Its high mobility and electrical stability enable reliable detection of the weak nerve action potential at a low power consumption. High-performance InSe FETs expand their promising applications in flexible and in situ real-time intelligent nerve action potential recording.

npj 2D Materials and Applications (2019)3:29; https://doi.org/10.1038/s41699-019-0110-x

\section{INTRODUCTION}

Two-dimensional (2D) materials have attracted intensive attention because of their unique electronic structure and transport properties. ${ }^{1-9}$ Among 2D materials, indium selenide (InSe), a layered semiconductor made of stacked layers of Se-In-Se atoms, holds great promise because of its high intrinsic mobility and moderate electronic band gap $(1.26 \mathrm{eV}) .^{10-12}$ Previous work has been performed to improve the mobility of InSe field-effect transistors (FETs) and other 2D material-based FETs. ${ }^{12-15}$ For instance, (1) Heterojunction structure, ${ }^{16}$ (2) high-k dielectric, ${ }^{1,13}$ (3) high-k encapsulation, ${ }^{17}$ and (4) Chemical and physical interface engineering ${ }^{15,18,19}$ have been used to enhance the carrier mobilities of 2D materials based FETs. However, in addition to the carrier mobility of FETs, electrical stability is another extremely important factor in ensuring device reliability in practical applications. Electrical instability is expressed mainly as threshold voltage shifts and carrier mobility degradation. ${ }^{20-22}$ Despite the considerable amount of theoretical and experimental research reported on 2D material-based devices, understanding of and improvements in their electrical stability are still in their infancy and remain a formidable challenge.

Earlier studies of InSe revealed that the performance of InSe FETs can be significantly influenced by the dielectric-2D semiconductor interface and the number of layers. ${ }^{13}$ At the dielectricInSe interface, in addition to the intrinsic acoustic phonon scattering of multilayer InSe, interfacial Coulomb impurities (Cls), surface roughness, surface chemical properties and surface polar phonon scattering from the dielectric can all impact the carrier transport in InSe FETs. Both the carrier mobility and electrical stability of FETs fundamentally depend on the state of the dielectric-semiconductor interface, and the mechanism is complex. It was found that charge impurity scattering has important impact on transistor performance. ${ }^{3}$ Ghatak et al. claimed that Coulomb potential from trapped charges in the substrate is the dominant source of disorder in $\mathrm{MoS}_{2}$ field-effect devices. ${ }^{23}$ Researchers have investigated the influence of trapped charges on $\mathrm{MoS}_{2}$ transistor performance. ${ }^{24,25}$ However, an effective, convenient, and inexpensive approach is needed for improving both field-effect mobility and electrical stability of transistors, to expand InSe FETs to practical applications-highly sensitive and reliable sensors-for instance, for neuron action potential detection. Neural signal transmission is fundamental to diverse human behaviors, such as learning, cognition, motor action, emotion, perception, and consciousness. ${ }^{26-30}$ Moreover, improper functioning or abnormal neural signals are associated with numerous neurological disorders, such as high paraplegia, epilepsy, and Parkinson's disease. ${ }^{31}$ Thus, highly sensitive and stable neural signal sensors capable of real-time and on-site detection are needed. ${ }^{32-34}$ High-performance InSe FETs are promising for realizing real-time, in situ neuron action potential detection, because of their high amplification factor and device flexibility when prepared on flexible substrates.

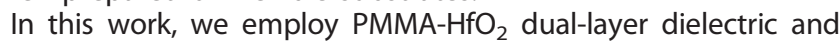
back-channel encapsulation to effectively improve the electrical

\footnotetext{
${ }^{1}$ Institute of Marine Science and Technology, Shandong University, 266237 Qingdao, China; ${ }^{2}$ Shenzhen Research Institute, Shandong University, 518057 Shenzhen, China; ${ }^{3}$ Center of Nanoelectronics and School of Microelectronics, Shandong University, 250100 Jinan, China; ${ }^{4}$ Department of Physiology, Shandong University School of Medicine, 250100 Jinan, China; ${ }^{5}$ National Research Center for Assisted Reproductive Technology and Reproductive Genetics, Shandong University, 250100 Jinan, China; ${ }^{6}$ Institute of Microelectronics and Beijing National Research Center for Information Science and Technology (BNRist), Tsinghua University, 10084 Beijing, China; ${ }^{7}$ State Key Laboratory of Crystal Materials, Shandong University, 250100 Jinan, Shandong, China and ${ }^{8}$ Institute for Advanced Interdisciplinary Research (iAIR), University of Jinan, 250022 Jinan, China Correspondence: Yu Zhang (yuzhang@sdu.edu.cn) or Tian-Ling Ren (RenTL@tsinghua.edu.cn) or Lin Han (hanlin@sdu.edu.cn)

These authors contributed equally: Jianfeng Jiang, Jingxin Li, Yutao Li
}

Received: 8 April 2019 Accepted: 4 July 2019

Published online: 30 July 2019 


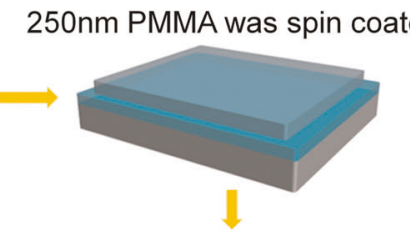

InSe micromechanical exfoliation

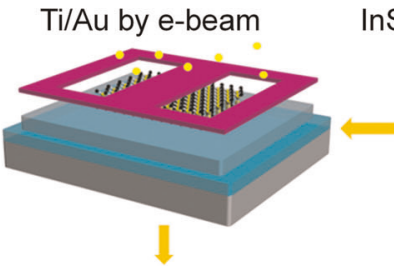

Shadow mask was removed
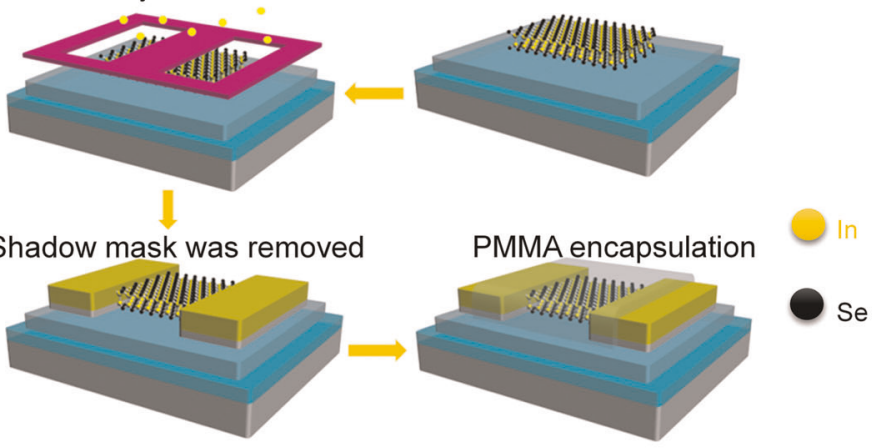

d

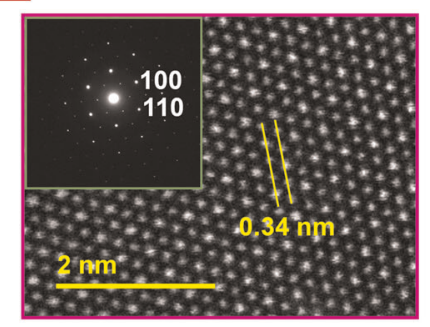

e

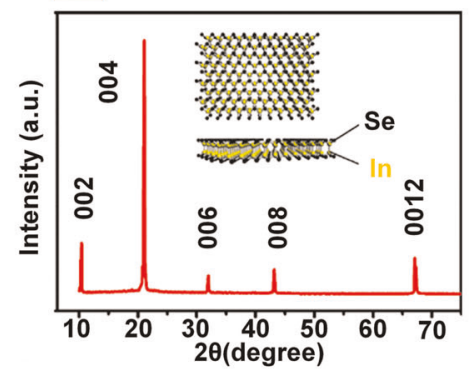

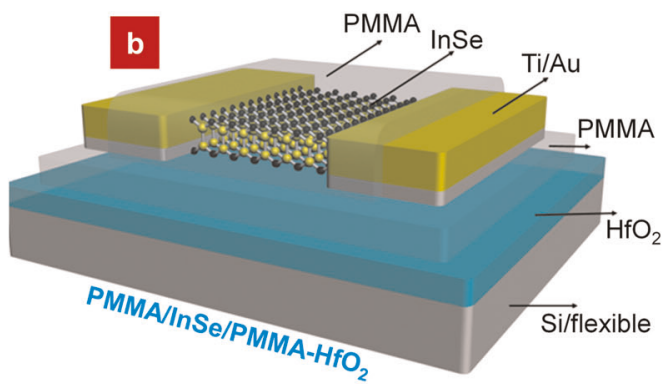

c

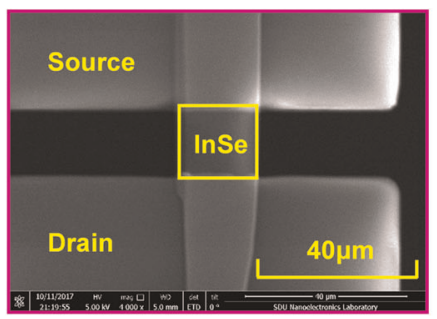

f

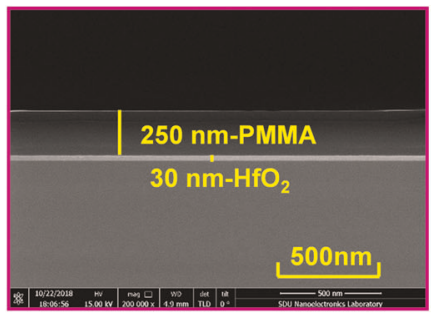

Fig. 1 Schematics of InSe FETs and their fabrication process. a The fabrication protocol of the PMMA/InSe/PMMA-HfO 2 FET. b Schematic diagram of a PMMA/InSe/PMMA-HfO 2 FET. c Flat SEM image of an InSe FET. d High-resolution TEM image of an InSe flake and a fast Fourier transformation (FFT) image. e XRD pattern of an InSe flake. Inset: Schematic diagram of the InSe crystal structure. $\mathbf{f}$ Cross-sectional SEM image of the InSe FET dielectric layer

stability of InSe while maintaining its high mobility $\left(\sim 1200 \mathrm{~cm}^{2} / \mathrm{N} \cdot \mathrm{s}\right)$ under normal ambient conditions. The time-dependent and stressdependent electrical stability was systematically analyzed. The hysteresis and threshold voltage shift in the working regime exhibit a negligible change ( $0.2 \mathrm{~V})$ over one month. Furthermore, density functional theory (DFT) was used to illustrate the basic physics and electrical stability mechanism of our newly configured InSe FETs. The calculation result shows that the increase in electron effective mass is relatively small when InSe forms the heterogeneous junction with PMMA compared to that for other dielectric substrates, which is consistent with the change in FET performance induced by different dielectric substrates. Based on the high electrical stability and field-effect mobility, InSe FETs allow us to conduct the real-time and in situ detection of frog sciatic action potential. The output signal of action potential was clean and traceable because of the transistors' stable electrical performance. This valuable method could be easily expanded to other 2D materials and could open up new opportunities for these materials in high-performance sensing applications.

\section{RESULTS AND DISCUSSION}

The fabrication of our transistors started with deposition of a $30 \mathrm{~nm} \mathrm{HfO} 2$ layer on a highly doped $\mathrm{Si}$ wafer by an atomic layer deposition (ALD) system at $150^{\circ} \mathrm{C}$ and spin-coating of a $250 \mathrm{~nm}$ PMMA layer on $\mathrm{HfO}_{2}$, which function as the dual-layer dielectric (Fig. 1a, b). Supplementary Fig. 1 presents schematic diagrams of other InSe FET structures. A typical scanning electron microscopy
(SEM) image of a multilayer InSe FET with a flexible PMMA and high dielectric constant $\mathrm{HfO}_{2}$ dual-layer dielectric together with PMMA back-channel encapsulation (PMMA/InSe/PMMA-HfO ${ }_{2}$ FET) is shown in Fig. 1c. The Raman spectrum (Supplementary Fig. 2a) of the multilayer InSe flakes shows peaks at 115, 178, and $228 \mathrm{~cm}^{-1}$, corresponding to the vibration modes of $A_{1}^{\prime}\left(\Gamma^{2}{ }_{1}\right)$, $\mathrm{E}^{\prime}\left(\Gamma_{3}{ }_{3}\right)-\mathrm{TO}$, and $\mathrm{A}_{1}{ }^{\prime}\left(\Gamma^{3}{ }_{1}\right) .^{35}$ The thickness of the InSe flake was determined by atomic force microscopy (AFM). Supplementary Fig. $2 b$ shows a typical surface shape graph and AFM step scan image of an InSe flake $(\sim 35 \mathrm{~nm})$. As shown in Fig. 1d, a highresolution STEM image with an inset obtained by reverse Fourier transform presents the perfect crystallinity of the InSe flake with a hexagonal lattice spacing of $3.4 \AA(0.34 \mathrm{~nm}))^{13}$ The chemical composition of the as-prepared InSe flake consists of indium and selenium at an atomic ratio of $\sim 1: 1$, as measured by energydispersive X-ray spectroscopy (EDS). The elemental distribution within the marked area was characterized by using the composition mapping operation, indicating the homogenous distribution of indium and selenium over the sheet (Supplementary Fig. 2c-e). In the XRD pattern of the InSe crystal, which can be found in Fig. $1 \mathrm{e}$, the five peaks correspond to (00I) $(I=2,4,6,8,12)$ without any other impurities, suggesting the high crystalline purity of the asprepared InSe crystal. The dual-layer structure shows smooth physical contact under SEM (Fig. 1f). To extract the accurate electron mobility, the capacitor structure of high-k dielectric materials was fabricated, and the capacitance was measured as shown in Supplementary Fig. 3. It can be seen that a very high capacitance of $C_{\mathrm{Ox}}=1200 \mathrm{nF} / \mathrm{cm}^{2}$ could be obtained. The contact 
a

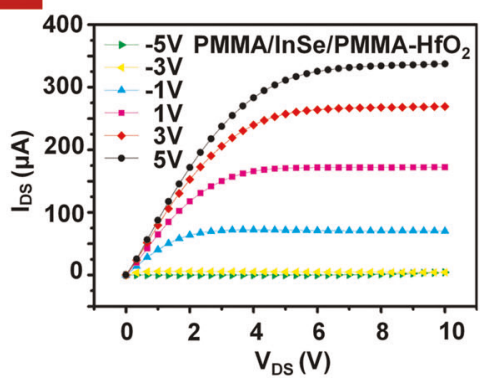

b

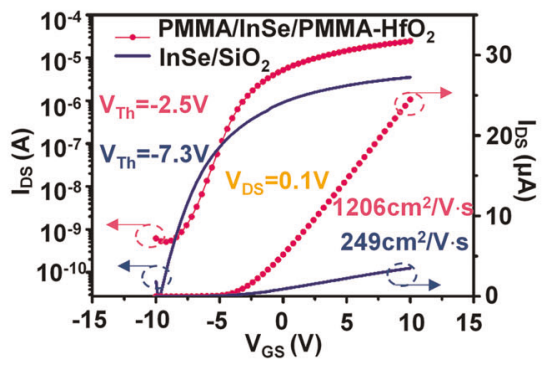

c

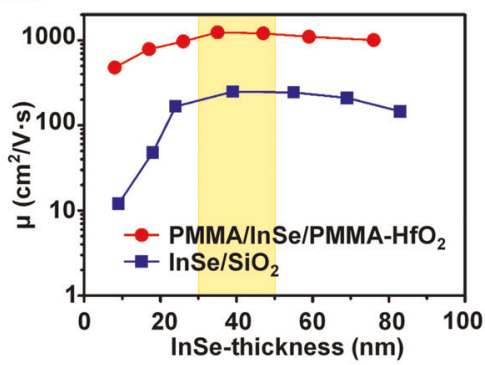

Fig. 2 Electrical characteristics and mobility of InSe FETs. a Output characteristics of a PMMA/InSe/PMMA-HfO 2 FET. The channel length and width are $21 \mu \mathrm{m}$ and $25 \mu \mathrm{m}$, respectively. $\mathbf{b}$ Transfer characteristics of PMMA/InSe/PMMA-HfO ${ }_{2}$ and InSe/SiO ${ }_{2} \mathrm{FETs}_{\text {at }} \mathrm{V}_{\mathrm{DS}}=0.1 \mathrm{~V}$. c Thicknessdependent field-effect mobilities of multilayer InSe FETs with PMMA/InSe/PMMA-HfO 2 and InSe/SiO ${ }_{2}$ configurations
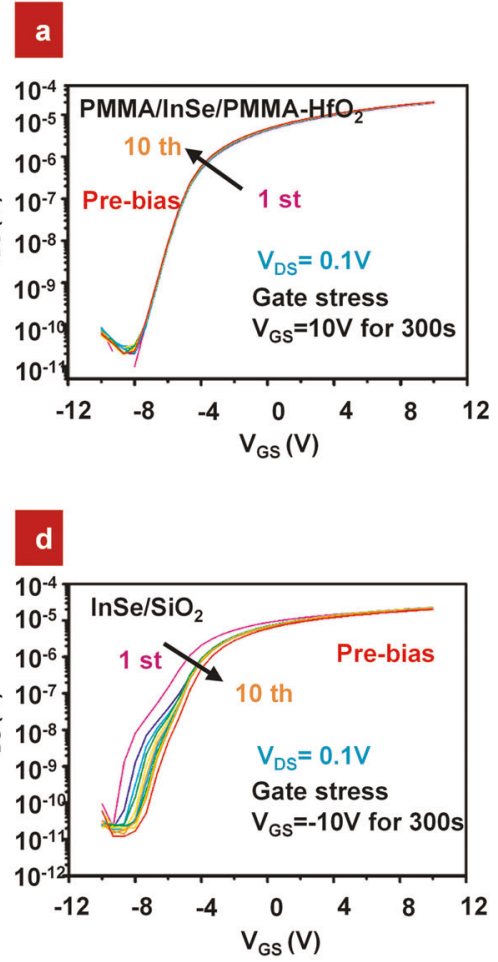

g

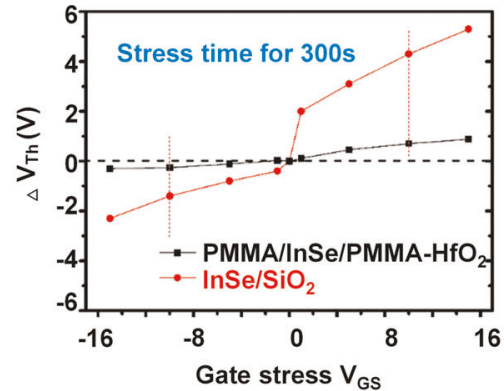

b

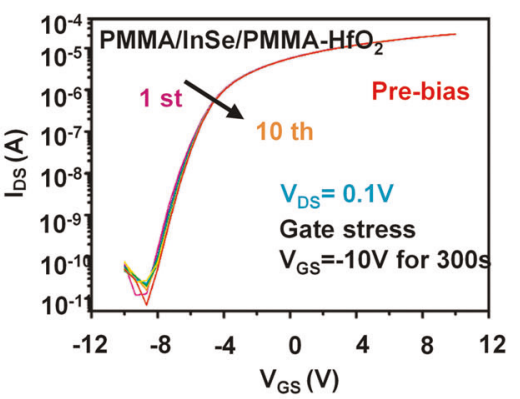

e

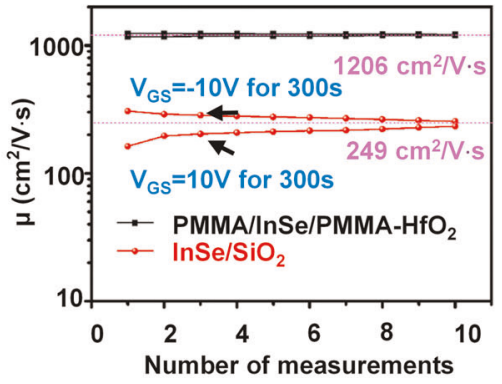

h

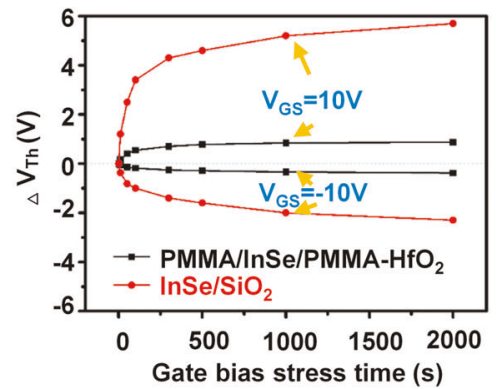

c

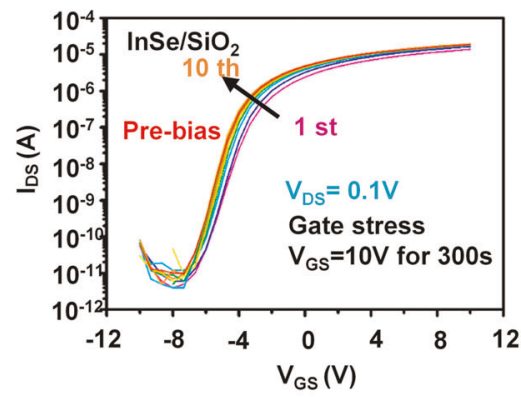

f

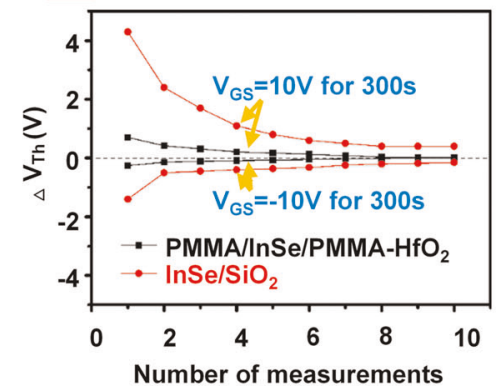

i

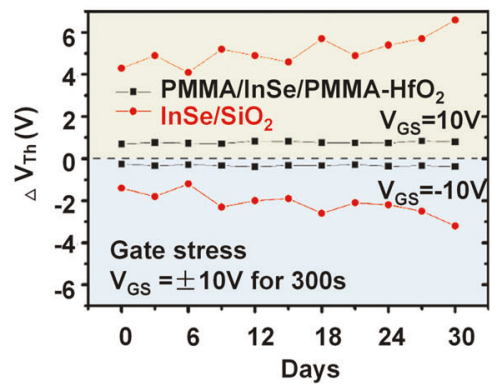

Fig. 3 Electrical stability of InSe FET. Transfer characteristics of $\mathbf{a}$, $\mathbf{b}$ a PMMA/InSe/PMMA-HfO $\mathrm{FET}_{2}$ and $\mathbf{c}$, $\mathbf{d}$ an InSe/SiO $\mathrm{FET}_{2} \mathrm{measured}$ before the gate bias stress (prestress) and 10 consecutive $I_{\mathrm{DS}}-V_{\mathrm{GS}}$ curves immediately after $\mathbf{a}, \mathbf{c} V_{\mathrm{GS}}=10 \mathrm{~V}$ and $\mathbf{b}, \mathbf{d} V_{\mathrm{GS}}=-10 \mathrm{~V}$ gate bias stress was applied for $300 \mathrm{~s}$. e Field-effect mobility of the PMMA/InSe/PMMA-HfO 2 FET and InSe/SiO 2 FET for each measurement in the liner regime following the gate bias stress. $f$ Threshold voltage shifts of the PMMA/InSe/PMMA-HfO $\mathrm{FET}_{2}$ and InSe/SiO $\mathrm{F}_{2} \mathrm{FET}$ for each measurement compared with the value of the pre-bias curves. $\mathbf{g}$ Threshold voltage shift with different gate stresses $(-15,-10,-5,-1,0,1,5,10,15 \mathrm{~V})$ for $300 \mathrm{~s} . \mathbf{h}$ Threshold voltage shift with different gate stress times $(0,10,50,100,300,500,1000$, and $2000 \mathrm{~s})$ at gate stress $V_{\mathrm{GS}}= \pm 10 \mathrm{~V}$. $\mathbf{i}$ Threshold voltage shift of the PMMA/InSe/PMMA-HfO $2 \mathrm{FET}$ and InSe/SiO 2 FET over 30 days 
angles for water on different substrates are shown in Supplementary Fig. 4, indicating that the PMMA film is more hydrophobic than the other oxidized dielectrics, which is conducive to improving interface conditions by suppressing carrier scattering from chemical impurities. To ensure clean contacts and avoid possible contamination from photolithography, the source and drain contacts, $\mathrm{Ti} / \mathrm{Au}(10 / 50 \mathrm{~nm})$, were defined by a shadow mask and deposited by electron-beam evaporation. Then, PMMA was spin-coated and baked to encapsulate the back-channel of the InSe FET. PMMA encapsulation can effectively retard water and oxygen molecules penetrating, which improves electrical stability of InSe FETs in air. The Raman peak and intensity did not change significantly during the 20-day test, indicating that InSe was stable for a relatively long time in the case of PMMA encapsulation. Its Raman spectrum is shown Supplementary Fig. 5.

The $I_{\mathrm{DS}}-V_{\mathrm{DS}}$ output characteristics and $I_{\mathrm{DS}}-V_{\mathrm{GS}}$ transfer characteristics of our multilayer InSe FETs were measured with an Agilent B2901A parameter analyzer under ambient conditions. For the output characteristics ( $35 \mathrm{~nm}-\mathrm{InSe}$ ), the $V_{\mathrm{DS}}$ is swept from 0 to $10 \mathrm{~V}$ while increasing $V_{\mathrm{GS}}$ from -5 to $5 \mathrm{~V}$ in increments of $2 \mathrm{~V}$ (Fig. 2a). For the transfer characteristics $(35 \mathrm{~nm}-\operatorname{lnSe})$, the $V_{\mathrm{GS}}$ is swept from -10 to $10 \mathrm{~V}$ at a $V_{D S}$ of $0.1 \mathrm{~V}$ (Fig. 2b). From the leastsquares fit, we extract the linear electron mobility $\mu$ in the linear regime from InSe FETs with different thicknesses using the following equation: $\mu=\left(\frac{L}{W C_{i} V_{D S}}\right)\left(\frac{d l_{D S}}{d V_{G S}}\right)$. The InSe FETs turned on at a negative gate bias, and the source/drain metal electrodes had ohmic contacts with the InSe channel layer, which enabled steady saturation currents. Characterization details for other structural devices are provided in the Supplementary Information (Supplementary Figs. 6, 7). However, more remarkably, PMMA/InSe/ $\mathrm{PMMA}^{-\mathrm{HfO}_{2}}$ FETs have better electrical properties than those of typical InSe/SiO 2 FETs or FETs with other structures. The small subthreshold slope (the following equation: $S S=\left(\frac{d \log \left(I_{D}\right)}{d V_{G}}\right)^{-1}$ ) of
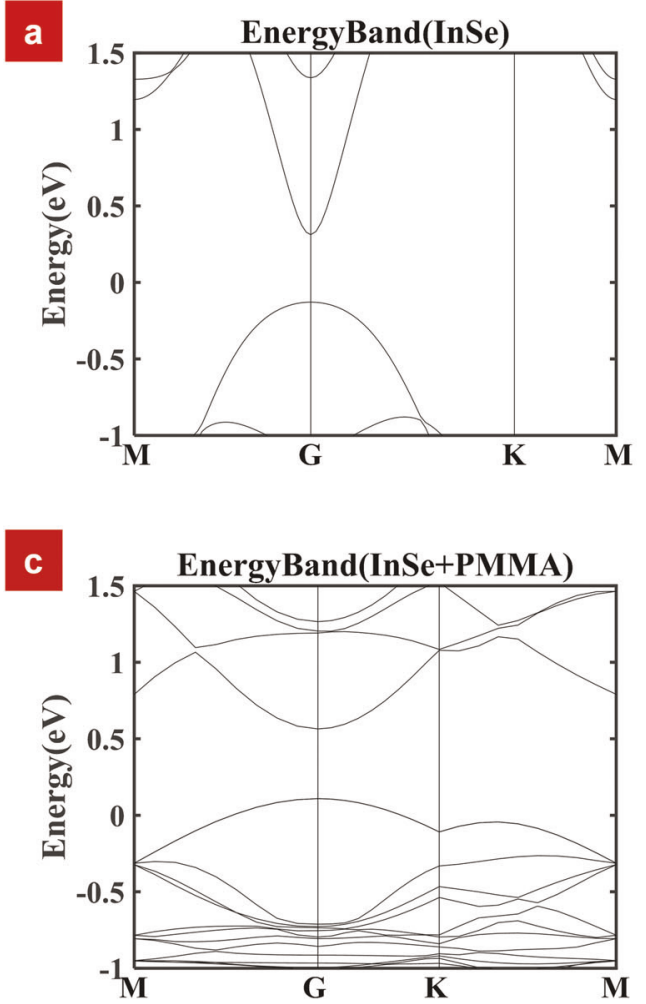

$260 \mathrm{mV} /$ decade and highest ON/OFF current modulation of $10^{7}$ extracted from the logarithmic plot indicate that the InSe transistor has a fast ON/OFF speed, which is an important factor for its electronic applications. A threshold voltage $V_{\text {th }}=-2.5 \mathrm{~V}$ and effective electron mobility of $1200 \mathrm{~cm}^{2} / \mathrm{V} \cdot \mathrm{s}$ in the working regime were extracted from the linear plot of transfer characteristics (Fig. 2b). We experimentally investigated the impact of InSe FETs with different thicknesses. As shown in Fig. 2c, the carrier scattering exhibits an InSe thickness-dependent impact on the mobilities of layered InSe FETs, but our PMMA/InSe/PMMA-HfO FETs show much less thickness dependence, which will effectively contribute to device consistency in practical applications. The mobility increases as the InSe layer thickness increases from $\sim 5 \mathrm{~nm}$ to $35 \mathrm{~nm}$ and then decreases as the channel thickness further increases for both $\mathrm{SiO}_{2} / \mathrm{InSe}$ and $\mathrm{PMMA} / \mathrm{InSe} / \mathrm{PMMA}-\mathrm{HfO}_{2}$ FETs. Subsequently, InSe films with a thickness of $\sim 35 \mathrm{~nm}$ were selected to build high-performance FETs.

Figure 3 shows the electrical stability behaviors in InSe transistors ( $35 \mathrm{~nm}-\mathrm{InSe}$ ). The most intuitive observation of electrical instability is the shift in the transfer curve during repeated transistor operations. During operation, the gate stress triggers interface defect states, which induce threshold voltage shifts. The PMMA/InSe/PMMA- $\mathrm{HfO}_{2}$ FETs feature a remarkable current reproducibility as the gate sweeps 10 times after a gate stress of $V_{\mathrm{GS}}= \pm 10 \mathrm{~V}$ (Fig. 3a, b), which matches its negligible hysteresis shown in Supplementary Fig. 7. In addition, compared to the PMMA/InSe/PMMA-HfO 2 FETs, the InSe/SiO ${ }_{2}$ FETs have a larger threshold voltage shift after gate stress (Fig. 3c, d). After the release of gate stress, the interface starts to return to its original state, and as a result, the transfer curve shifted back close to its curve sweep before the application of gate stress. The mobility of $\mathrm{PMMA} / \mathrm{InSe} / \mathrm{PMMA}-\mathrm{HfO}_{2}$ is less sensitive to the gate bias stress than that of $\operatorname{lnSe} / \mathrm{SiO}_{2}$ and $\operatorname{InSe} / \mathrm{HfO}_{2}$, as shown in Fig. 3e and Supplementary Fig. 8. Threshold voltages derived from the transfer plots show a maximum shift of $\sim 0.6 \mathrm{~V}$ from the PMMA/
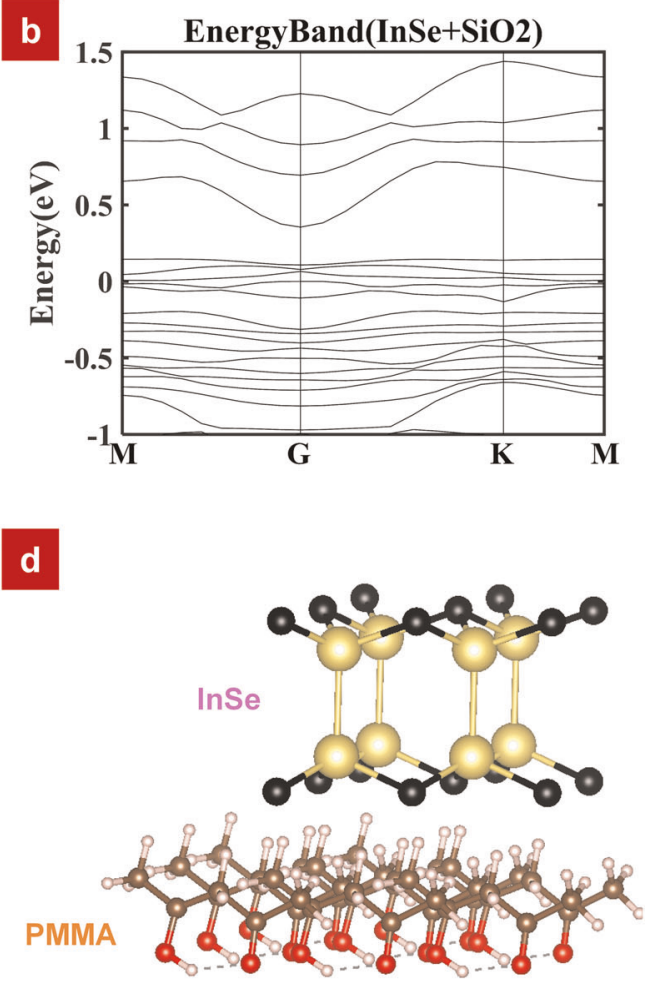

Fig. 4 First-principles calculations for different interface conditions. The band structures corresponding to $\mathbf{a}$ intrinsic $\operatorname{lnSe}, \mathbf{b}$ InSe/SiO ${ }_{2}$, and $\mathbf{c}$ InSe/PMMA. d Schematic diagram of the InSe/PMMA structure 
InSe/PMMA-HfO 2 FETs in Fig. 3f, which is less than $1 / 10$ of that of the $\mathrm{InSe} / \mathrm{SiO}_{2}$ FETs. More interface defects were triggered or induced with increased gate stress strength and time, which caused a larger threshold voltage shift (Fig. $3 g$, h). After 5 min, the threshold voltage shifts reach a steady state because most defect states participate in electrical activity in a short time, and few defects states become activated with stress strength and time. After over 30 days of storage at an ambient temperature, the threshold voltage shift in Fig. $3 \mathrm{i}$ remained at $\sim 0.5 \mathrm{~V}$ under the same gate stress condition, and the threshold voltage shift of the transistor with the $\mathrm{SiO}_{2}$ dielectric was in the range of $\sim 4-7 \mathrm{~V}$. These results show that compared to other structural devices, our $\mathrm{PMMA} / \mathrm{InSe} / \mathrm{PMMA} \mathrm{HfO}_{2}$ FETs have superior electrical stability. In addition, InSe FETs with different encapsulation layers were fabricated to compare their effects on electrical properties and stability of the device. PMMA, $\mathrm{Al}_{2} \mathrm{O}_{3}, \mathrm{HfO}_{2}$ were used as FETs encapsulation layer, the electrical properties were systematically studied including mobility, current on/off ratio, hysteresis, and threshold voltage shift. PMMA encapsulated InSe FET had higher field-effect mobility, smaller hysteresis, and lower threshold voltage shift at the same test condition, and the results are shown in Supplementary Fig. 9 and Table S1.

According to the electronic measurement shown above, spincoating with PMMA clearly causes a significant improvement in device mobility, and the choice of different dielectric materials can also affect the device mobility. Screen of disorder may be an important reason according to previous study on dual layer PMMA to improve the mobility of $2 \mathrm{D}$ material. ${ }^{13,36}$ Furthermore, the improvement in mobility and stability of the transistor is key to nerve action signal detection. To further study the underlying

Table 1. DFT calculation of the relative effective masses for electrons under different conditions

\begin{tabular}{llll}
\hline Structures & $\begin{array}{l}\text { Relative mass at the bottom } \\
\text { of the conduction band }\end{array}$ & Fermi level & $\begin{array}{l}\text { Lattice } \\
\text { mismatch }\end{array}$ \\
\hline Intrinsic InSe & 1 (normalization) & $-14.2 \mathrm{eV}$ & $\mathrm{NA}$ \\
InSe/PMMA & 1.56 & $-14.2 \mathrm{eV}$ & $\mathrm{NA}$ \\
$\operatorname{In} \mathrm{Se} / \mathrm{HfO}_{2}$ & 1.96 & $-14.8 \mathrm{eV}$ & $2.02 \%$ \\
$\ln \mathrm{Se} / \mathrm{Al}_{2} \mathrm{O}_{3}$ & 3.85 & $-14.65 \mathrm{eV}$ & $1.17 \%$ \\
$\ln \mathrm{Se} / \mathrm{SiO}_{2}$ & 4.17 & $-14.1 \mathrm{eV}$ & $3.25 \%$ \\
\hline
\end{tabular}

physical mechanism of the device, first-principles calculations were performed using the Vienna Ab initio Simulation Package (VASP) code based on DFT. ${ }^{37,38}$ The band structure and electron effective mass of InSe on different dielectric materials $\left(\mathrm{HfO}_{2}, \mathrm{Al}_{2} \mathrm{O}_{3}\right.$, $\mathrm{SiO}_{2}$ ) were studied. In addition, PMMA was placed on the InSe supercell to investigate the influence of PMMA. The calculation details are shown in the Methods section (shown in Supplementary Fig. 10). The band structures of intrinsic InSe, the InSe/SiO heterojunction and the InSe/PMMA heterojunction are shown in Fig. $4 a-c$, respectively. In general, the curvature of the band reflects the electron effective mass, and a lower effective mass usually indicates higher mobility. Clearly, the curvature of the InSe band decreases significantly after the formation of the heterojunction. Detailed calculation results are shown in Table 1. The increase in the electron effective mass was relatively small when InSe formed the heterogeneous junction with PMMA compared to other dielectric substrates, which was consistent with our hypothesis and experimental results. In addition, the introduction of PMMA did not cause obvious changes in the Fermi energy level, which means the Fermi Level of InSe in the InSe/PMMA structure is the same as that in pure InSe according to the calculation results. However, the Fermi Level of InSe in InSe/ $/ \mathrm{Al}_{2} \mathrm{O}_{3}$, InSe/ $/ \mathrm{HfO}_{2}$, and $\mathrm{InSe} / \mathrm{SiO}_{2}$ structures changed according to DFT calculation results, which further indicated that PMMA has little influence on the electronic properties of InSe. In addition, PMMA is flexible and does not bring any lattice mismatch. The hydrophobic property of PMMA suppressed the chemical impurities of hydroxyl groups and absorbed water molecules at the oxidized dielectric (the water contact angles of PMMA and other dielectric materials are shown in Supplementary Fig. 4). These series of simulation results, together with the material characterization results for PMMA, provided a firm explanation for the previously observed experimental phenomena.

Significantly, we summarize the electrical properties of 2D FETs from the literature obtained using different approaches in Table 2. Clearly, compared to the other 2D FETs, our PMMA/InSe/PMMA$\mathrm{HfO}_{2}$ FETs show better electrical performance and stability, which further confirmed the advantage of our technology. Encouraged by the high performance of the InSe FETs, we applied them to sciatic nerve action potential recording (Fig. 5c). Before the actual recording of frog sciatic nerve action potential, we assessed the transistor response to weak electrical signals by applying a sequence of gate voltage $V_{G S}$ pulses with a peak value of

Table 2. Summary of 2D FETs in different reports

\begin{tabular}{|c|c|c|c|c|c|c|c|}
\hline $\mathrm{MoS}_{2}$ & $\mathrm{HfO}_{2}$ & - & $\sim-3.5$ & 217 & $\sim 10^{6}$ & - & 1 \\
\hline $\mathrm{MoS}_{2}$ & $\mathrm{SiO}_{2}$ & $\sim 20$ & $\sim 5$ & 25.7 & $\sim 10^{5}$ & $\sim 15$ & 20 \\
\hline $\mathrm{MoS}_{2}$ & $\mathrm{SiO}_{2}$ & $\sim 25$ & $\sim-15$ & 10 & - & - & 21 \\
\hline $\mathrm{WS}_{2}$ & $\mathrm{SiO}_{2}$ & - & $\sim 20$ & $\sim 45$ & $\sim 10^{7}$ & - & 41 \\
\hline GeP & $\mathrm{SiO}_{2}$ & - & $\sim-25$ & 7.5 & $\sim 10^{5}$ & - & 42 \\
\hline $\ln -\operatorname{InSe}$ & $\mathrm{SiO}_{2}$ & $>10$ & $\sim-20$ & 3700 & $\sim 10^{7}$ & - & 15 \\
\hline $\mathrm{SnS}_{2}$ & $\mathrm{SiO}_{2}$ & - & $\sim 10$ & $\sim 50$ & $\sim 10^{7}$ & - & 43 \\
\hline InSe & $\mathrm{PMMA} / \mathrm{Al}_{2} \mathrm{O}_{3}$ & - & $\sim-3$ & 1055 & $\sim 10^{7}$ & - & 13 \\
\hline
\end{tabular}



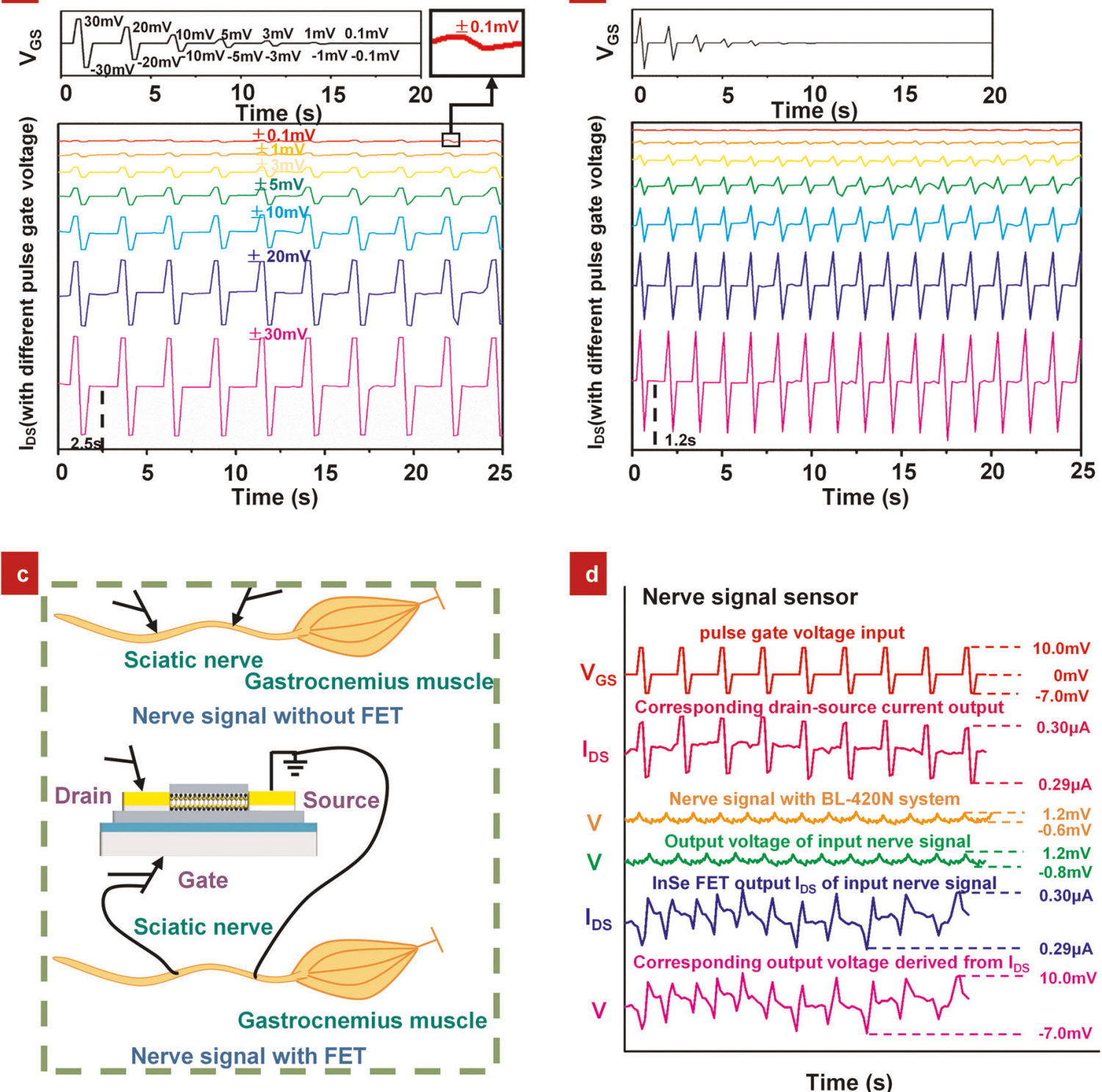

Fig. 5 Sciatic nerve signal detection. a, b Drain-source current $I_{D s}$ with different gate voltage pulses $(0.1,1,3,5,10,20,30 \mathrm{mV}$ for $1 \mathrm{~s}$ and $3 \mathrm{~s}$ cycles). c Schematic diagram of structures with/without PMMA/InSe/PMMA-HfO ${ }_{2}$ FETs for detecting sciatic nerve signals. d Sciatic nerve signal detection with/without PMMA/InSe/PMMA-HfO 2 FETs

$0.1-30 \mathrm{mV}$ at intervals of $3 \mathrm{~s}$ and $1 \mathrm{~s}$, recording the source/drain current $I_{\mathrm{DS}}$ at $V_{\mathrm{DS}}=0.1 \mathrm{~V}$ (Fig. $5 \mathrm{a}, \mathrm{b}$ ). The $I_{\mathrm{DS}}$ is linearly dependent on the input $V_{G S}$ defined by the formula $I_{D S}=$ $\mu C_{\mathrm{ox}} \frac{W}{L}\left(V_{\mathrm{GS}}-V_{\mathrm{T}}\right) V_{\mathrm{DS}}$ in the linear regime with $V_{T}=-2.5 \mathrm{~V}$. The correlation between the input $V_{G S}$ pulse and recorded $I_{D S}$ was experimentally derived and was consistent with the value theoretically calculated from the formula in linear regime (Supplementary Fig. 11). The sciatic nerve of a live frog was carefully dissected along the edge of the vertebral column and the sciatic groove located between the biceps femoris and semimembranosus muscle. During this entire process, the sciatic nerve was constantly dipped or soaked in Ringer's solution to keep the nerve alive. For the recording of the biphasic action potential, the gate and source electrodes were connected to the middle part of the sciatic nerve, and the source-drain current $I_{\mathrm{DS}}$ was recorded by a semiconductor parameter analyzer. The sciatic nerve was stimulated by intermittent clamping with tweezers, and its nerve action potential was applied to the gate electrode of the FET, which produced the corresponding source-drain current $\mathrm{I}_{\mathrm{DS}}$ (Fig. $5 \mathrm{~d})$. In order to avoid the stimulus artifact to interfere our recorded electrical signal, we used mechanical stimulation by tweezers clamping rather than stimulator. We found that by once clamping, one electrical signal appeared, which was accompanied by a single muscle twitch. Therefore, we think the signal recorded in our study definitely is compound action potential (CAP). Beside, as we know that typical sciatic nerve action potential is a characteristic biphasic potential. When both electrodes are placed on the surface of an axon, nerve is stimulated, and the nerve impulse produces a characteristic biphasic potential. The wave we recorded is as same as the typical one. (Mechanism diagram of sciatic nerve detection in Supplementary Fig. 12) Except nerve action potential, no gate bias was applied, since InSe transistor has threshold voltage of $-2.5 \mathrm{~V}$ and turns on at $V_{\mathrm{GS}}=0 \mathrm{~V}$. At $V_{\mathrm{DS}}=$ $0.1 \mathrm{~V}$, corresponding $\mathrm{I}_{\mathrm{Ds}}$ produced by nerve action potential was recorded. Finally, the nerve action potential was derived from the recorded $I_{D S}$ according to the correlation shown in Supplementary 
Fig. 11. Compared to the conventional BL-420N measurement and direct measurement with the Agilent B2901A parameter analyzer, our InSe transistor recorded a much more distinct signal corresponding to the frog's leg action (Fig. 5d and Video in Supplementary Fig. 13) because the transistors' stable electrical performance. In addition, InSe FETs have great potential to be fabricated into flexible devices for in situ real-time recording. Supplementary Fig. 14 shows flexible PMMA/InSe/PMMA-HfO FETs on a polyimide substrate, even though more detailed work is needed to realize high-performance InSe FETs.

In conclusion, a flexible/hydrophobic PMMA and high dielectric constant $\mathrm{HfO}_{2}$ dual-layer together with PMMA back-channel encapsulation allows InSe FETs to achieve both high field-effect mobility and electrical stability. The threshold voltage shift was maintained at $0.6 \mathrm{~V}$ after 30 days of storage under normal ambient conditions, while the field-effect mobility of the InSe FET was maintained at $1200 \mathrm{~cm}^{2} / \mathrm{N} \cdot \mathrm{s}$. The PMMA layer used as a dielectric eliminated the lattice mismatch issue and did not cause obvious changes in the Fermi energy level of InSe. In addition, the hydrophobic property of PMMA suppressed the chemical impurities of hydroxyl groups and absorbed water molecules at the oxidized dielectric. The back-channel encapsulated PMMA protects InSe FETs from water and oxygen molecules influence at channel interface in the air. Highly stable InSe FETs with high mobility enabled real-time and in situ detection of sciatic nerve signals. This work revealed the important role of device electric stability in 2D materials, provides a valuable method to improve the electrical performance of 2D material-based FETs and may boost their practical applications, for instance, in the fields of neuron science, neurological diseases, and bio-molecules detection based diseases diagonosis.

\section{METHODS}

Device fabrication

Highly doped p-type silicon substrate was cleaned by buffered oxide etching (BOE) solution. ALD of $30 \mathrm{~nm} \mathrm{HfO}$ on the substrate was performed at $200^{\circ} \mathrm{C}$ at a growth rate of $1.1 \AA$ A/cycle. The $250 \mathrm{~nm}$ PMMA (950 K) layer (4000 rpm for $2 \mathrm{~min}$ ) was spin-coated onto the substrate with $30 \mathrm{~nm} \mathrm{HfO}_{2}$ and then baked at $170^{\circ} \mathrm{C}$ for $0.5 \mathrm{~h}$ in air. Layered InSe flakes were mechanically exfoliated from bulk InSe crystals and transferred to the prepared substrate using Scotch tape. To ensure clean contacts and avoid possible contamination from photolithography, the source and drain contacts, Ti/Au (10/50 $\mathrm{nm}$ ), were defined by a shadow mask and deposited by electron-beam evaporation ( $2 \AA / \mathrm{s})$. To prevent cracking of the metal, a copper sleeve was used during the evaporation process to shield from heat radiation. Finally, PMMA was spin-coated and baked to encapsulate the back-channel of the InSe FETs. Electrical characterization was carried out with an Agilent B2901A parameter analyzer under ambient conditions.

\section{First-principles calculation}

Before starting the calculations, the supercell of intrinsic InSe and a substrate with a lattice mismatch less than $4 \%$ was chosen (lattice mismatch is shown in Table 1). In addition, when considering the effect of PMMA, the supercell of intrinsic InSe was built first, and the PMMA molecules were placed on the supercell to form the heterostructure. The VASP code based on DFT was used to calculate the bandgap and effective masses of three material systems. ${ }^{37-39}$ The generalized gradient approximation (GGA) with the Perdew Burke Ernzerhof (PBE) function was used. ${ }^{39}$ Brillouin zone sampling was performed using a Monkhorst-Pack special kpoint grid. ${ }^{40}$ The grids for the k-point sampling were $4 \times 4 \times 1$ for the primitive cell. The force tolerance in structure relaxation was $0.005 \mathrm{eV} / \mathrm{nm}$, the energy tolerance was $10^{-4} \mathrm{eV}$, and the energy cutoff was $400 \mathrm{eV}$.

\section{Sciatic nerve signal detection}

The frog was held on the left hand with cotton or cloth. The brain and spinal cord were destroyed by pitching. After pitching, the head, viscera, and skin were removed from the body. With the help of a glass dissecting needle, the sciatic nerve was carefully dissected along the edge of the vertebral column and the sciatic groove located between the biceps femoris and semimembranosus muscle. During this entire process, the sciatic nerve should be constantly dipped or soaked in Ringer's solution, and it should not be touched by hand or with a metallic object. After stimulating the upper part of the sciatic nerve, the biphasic action potential and contraction of the gastrocnemius muscle were observed.

\section{DATA AVAILABILITY}

Data are available on reasonable request from the authors.

\section{ACKNOWLEDGEMENTS}

This research was supported by the National Key R\&D Plan of China (Grant No. 2017YFB0405400, 2016YFA0200400, 2015CB352101), Natural Science Foundation for Yung Scientists of China (Grant No. 61701282, 51861145202, 61574083, 61434001), Free Exploration Project for Basic Research of Shenzhen (Grant No. JCYJ20170307093106023), Natural Science Foundation for Distinguished Young Scientist of Shandong Province (Grant No. JQ201814), the Fundamental Research Fund of Shandong University (Grant No. 2017JC020), and Open Project of National Research Center for Assisted Reproductive Technology and Reproductive Genetics, Shandong University.

\section{AUTHOR CONTRIBUTIONS}

L.H. and T.R. conceived the project. J.J. developed device fabrication method and performed electric measurement. Y.L., L.L. and Y.T. carried out DFT calculations and modeling. J.J. performed materials characterization with help of J.D. and H.L., J.L., Z.Z. and H.Z. dissected the sciatic nerve of frog and performed sciatic nerve signal detection. L.H., T.L.R., J.J., X.F., H.L., Q.L. and Y.Z. performed theoretical analysis. L.H. and J.J. wrote the paper and all authors commented on it.

\section{ADDITIONAL INFORMATION}

Supplementary information accompanies the paper on the npj 2D Materials and Applications website (https://doi.org/10.1038/s41699-019-0110-x).

Competing interests: The authors declare no competing interests.

Publisher's note: Springer Nature remains neutral with regard to jurisdictional claims in published maps and institutional affiliations.

\section{REFERENCES}

1. Radisavljevic, B., Radenovic, A., Brivio, J., Giacometti, V. \& Kis, A. Single-layer MoS transistors. Nat. Nanotechnol. 6, 147-150 (2011).

2. Wang, Q. H., Kalantar-Zadeh, K., Kis, A., Coleman, J. N. \& Strano, M. S. Electronics and optoelectronics of two-dimensional transition metal dichalcogenides. Nat. Nanotechnol. 7, 699-712 (2012).

3. Radisavljevic, B. \& Kis, A. Mobility engineering and a metal-insulator transition in monolayer $\mathrm{MoS}_{2}$. Nat. Mater. 12, 815-820 (2013).

4. Deng., Y. et al. Gate-tunable room-temperature ferromagnetism in twodimensional $\mathrm{Fe}_{3} \mathrm{GeTe}_{2}$. Nature 563, 94-99 (2018).

5. Zhang, Y., Tan, Y.-W., Stormer, H. L. \& Kim, P. Experimental observation of the quantum Hall effect and Berry's phase in graphene. Nature 438, 201-204 (2005).

6. Du, X., Skachko, I., Duerr, F., Luican, A. \& Andrei, E. Y. Fractional quantum Hall effect and insulating phase of Dirac electrons in graphene. Nature 462, 192-195 (2009).

7. Desai, S. B. et al. $\mathrm{MoS}_{2}$ transistors with 1-nanometer gate lengths. Science 354, 99-102 (2016).

8. Cui, X. et al. Multi-terminal transport measurements of $\mathrm{MoS}_{2}$ using a van der Waals heterostructure device platform. Nat. Nanotech. 10, 534-540 (2015).

9. Li, L. et al. Black phosphorus field-effect transistors. Nat. Nanotech. 9, 372-377 (2014).

10. Bandurin, D. A. et al. High electron mobility, quantum Hall effect and anomalous optical response in atomically thin InSe. Nat. Nanotech. 12, 223-227 (2017).

11. Lei, S. et al. Evolution of the electronic band structure and efficient photodetection in atomic layers of InSe. ACS Nano 8, 1263-1272 (2014).

12. Mudd, G. W. et al. Tuning the bandgap of exfoliated InSe nanosheets by quantum confinement. Adv. Mater. 25, 5714-5718 (2013).

13. Feng, W., Zheng, W., Cao, W. \& Hu, P. Back gated multilayer InSe transistors with enhanced carrier mobilities via the suppression of carrier scattering from a dielectric interface. Adv. Mater. 26, 6587-6593 (2014). 
14. Sucharitakul, S. et al. Intrinsic electron mobility exceeding $10^{3} \mathrm{~cm} 2 / \mathrm{Vs}$ in multilayer InSe FETs. Nano Lett. 15, 3815-3819 (2015).

15. Li, M. et al. High mobilities in layered inse transistors with indium-encapsulationinduced surface charge doping. Adv. Mater. 30, 1803690 (2018).

16. Lee, G. et al. Highly stable, dual-gated $\mathrm{MoS}_{2}$ transistors encapsulated by hexagonal boron nitride with gate-controllable contact, resistance, and threshold voltage. ACS Nano 9, 7019-7026 (2015).

17. Kim, S. et al. Enhanced carrier mobility of multilayer $\mathrm{MoS}_{2}$ thin-film transistors by $\mathrm{Al}_{2} \mathrm{O}_{3}$ encapsulation. Appl. Phys. Lett. 109, 152101 (2016).

18. $\mathrm{Yu}, \mathrm{Z}$. et al. Towards intrinsic charge transport in monolayer molybdenum disulfide by defect and interface engineering. Nat. Commun. 5, 5290 (2014).

19. Ho, P. et al. High-mobility InSe transistors: the role of surface oxides. ACS nano 11, 7362-7370 (2017).

20. Cho, K. et al. Electric stress-induced threshold voltage instability of multilayer $\mathrm{MoS}_{2}$ field effect transistors. ACS nano 7, 7751-7758 (2014)

21. Late, D. J. et al. Hysteresis in single-layer $\mathrm{MoS}_{2}$ field effect transistors. ACS nano 6, 5635-5641 (2012).

22. Feng, W. et al. Gate modulation of threshold voltage instability in multilayer inse field effect transistors. ACS Appl. MAter. Interface 7, 26691-26695 (2015).

23. Ghatak, S., Pal, A. N. \& Ghosh, A. Nature of electronic states in atomically thin $\mathrm{MoS}_{2}$ field-effect transistors. ACS Nano. 5, 7707-7712 (2011).

24. Stanford, M. G. et al. Lithographically patterned metallic conduction in singlelayer $\mathrm{MoS}_{2}$ via plasma processing. npj 2D Mater. Appl. 3, 13 (2019).

25. Ouyang, B. et al. Tunable phase stability and contact resistance of monolayer transition metal dichalcogenides contacts with metal. npj 2D Mater. Appl. 2, 13 (2018).

26. Silberberg, D. et al. Brain and other nervous system disorders across the lifespanglobal challenges and opportunities. Nature 527, S151-S154 (2015).

27. LeDoux, J. et al. Surviving threat: neural circuit and computational implications of a new taxonomy of defensive behavior. Nat. Rev. Neurosci. 19, 269-282 (2018).

28. Kang, S. K. et al. Bioresorbable silicon electronic sensors for the brain. Nature 530, 71-76 (2016).

29. Bedrosian, T. A. et al. Early life experience drives structural variation of neural genomes in mice. Science 359, 1395-1399 (2018).

30. Bryson, J. B. et al. Optical control of muscle function by transplantation of stem cell-derived motor neurons in mice. Science 344, 94-97 (2014).

31. Bouton, C. E. et al. Restoring cortical control of functional movement in a human with quadriplegia. Nature 533, 247-250 (2016).

32. Tybrandt, K. et al. High-density stretchable electrode grids for chronic neural recording. Adv. Mater. 30, 1706520 (2018).
33. Rivnay et al. Next-generation probes, particles, and roteins for neural interfacing. Sci. Adv. 3, e1601649 (2017).

34. Kim, D. et al. Epidermal electronics. Science 333, 838-843 (2011).

35. Yang, Z. et al. Wafer-scale synthesis of high-quality semiconducting twodimensional layered InSe with broadband photoresponse. ACS nano 11, 4225-4236 (2017)

36. Bao, W. et al. High mobility ambipolar $\mathrm{MoS}_{2}$ field-effect transistors: substrate and dielectric effects. Appl. Phys. Lett. 102, 042104 (2013).

37. Perdew, J. P. et al. Atoms, molecules, solids, and surfaces: applications of the generalized gradient approximation for exchange and correlation. Phys. Rev. B 46, 6671 (1992).

38. Kresse, G. et al. Efficient iterative schemes for ab initio total-energy calculations using a plane-wave basis set. Phys. Rev. B 54, 11169 (1996).

39. Kresse, G. et al. Vienna Ab-initio Simulation Package (VASP): the Guide. Universität Wien, VASP-Guide (2002).

40. Perdew, J. P. et al. Ernzerhof, generalized gradient approximation made simple. Phys. Rev. Lett. 77, 3865 (1996).

41. Aji, A. S. et al. High mobility $W_{2}$ transistors realized by multilayer graphene electrodes and application to high responsivity flexible photodetectors. Adv. Mater. 27, 1703448 (2017).

42. Li, L. et al. 2D GeP: an unexploited low-symmetry semiconductor with strong inplane anisotropy. Adv. Mater. 30, 1706771 (2018).

43. Song, H. S. et al. High-performance top-gated monolayer $\mathrm{SnS}_{2}$ field-effect transistors and their integrated logic circuits. Nanoscale 5, 9666-9670 (2013).

Open Access This article is licensed under a Creative Commons Attribution 4.0 International License, which permits use, sharing, adaptation, distribution and reproduction in any medium or format, as long as you give appropriate credit to the original author(s) and the source, provide a link to the Creative Commons license, and indicate if changes were made. The images or other third party material in this article are included in the article's Creative Commons license, unless indicated otherwise in a credit line to the material. If material is not included in the article's Creative Commons license and your intended use is not permitted by statutory regulation or exceeds the permitted use, you will need to obtain permission directly from the copyright holder. To view a copy of this license, visit http://creativecommons. org/licenses/by/4.0/.

(c) The Author(s) 2019 